\title{
First Limit on the Direct Detection of Lightly Ionizing Particles for Electric Charge as Low as $e / 1000$ with the Majorana Demonstrator
}

S. I. Alvis, ${ }^{1}$ I. J. Arnquist, ${ }^{2}$ F. T. Avignone III, ${ }^{3,4}$ A. S. Barabash, ${ }^{5}$ C. J. Barton, ${ }^{6}$ F. E. Bertrand,${ }^{4}$ V. Brudanin, ${ }^{7}$ M. Busch, ${ }^{8,9}$ M. Buuck, ${ }^{1}$ T. S. Caldwell, ${ }^{10,9}$ Y-D. Chan, ${ }^{11}$ C. D. Christofferson, ${ }^{12}$ P.-H. Chu, ${ }^{13}$ C. Cuesta, ${ }^{1,}$ J. A. Detwiler, ${ }^{1}$ C. Dunagan, ${ }^{12}$ Yu. Efremenko, ${ }^{14,4}$ H. Ejiri, ${ }^{15}$ S. R. Elliott, ${ }^{13}$ T. Gilliss, ${ }^{10,9}$ G. K. Giovanetti, ${ }^{16}$ M. P. Green,,${ }^{17,9,4}$ J. Gruszko, ${ }^{18}$ I. S. Guinn, ${ }^{1}$ V. E. Guiseppe, ${ }^{3}$ C. R. Haufe, ${ }^{10,9}$ L. Hehn, ${ }^{11}$ R. Henning, ${ }^{10,9}$ E. W. Hoppe, ${ }^{2}$ M. A. Howe, ${ }^{10,9}$ S. I. Konovalov, ${ }^{5}$ R. T. Kouzes, ${ }^{2}$ A. M. Lopez, ${ }^{14}$ R. D. Martin, ${ }^{19}$ R. Massarczyk,${ }^{13, \dagger}$ S. J. Meijer, ${ }^{10,9}$ S. Mertens,${ }^{20,21}$ J. Myslik, ${ }^{11}$ C. O’Shaughnessy, ${ }^{10,9, \#}$ G. Othman, ${ }^{10,9}$ W. Pettus, ${ }^{1}$ A. W. P. Poon, ${ }^{11}$ D. C. Radford, ${ }^{4}$ J. Rager,${ }^{10,9}$ A. L. Reine, ${ }^{10,9}$ K. Rielage, ${ }^{13}$ R. G. H. Robertson, ${ }^{1}$ N. W. Ruof, ${ }^{1}$ B. Shanks, ${ }^{4}$ M. Shirchenko, ${ }^{7}$ A. M. Suriano, ${ }^{12}$ D. Tedeschi, ${ }^{3}$ R. L. Varner,${ }^{4}$ S. Vasilyev, ${ }^{7}$ K. Vorren,${ }^{10,9}$ B. R. White,${ }^{13}$ J. F. Wilkerson, ${ }^{10,9,4}$ C. Wiseman, ${ }^{3}$ W. Xu, ${ }^{6}$ E. Yakushev, ${ }^{7}$ C.-H. Yu, ${ }^{4}$ V. Yumatov, ${ }^{5}$ I. Zhitnikov, ${ }^{7}$ and B. X. Zhu ${ }^{13}$

(MAJORANA Collaboration)

\author{
${ }^{1}$ Center for Experimental Nuclear Physics and Astrophysics, and Department of Physics, \\ University of Washington, Seattle, Washington 98195, USA \\ ${ }^{2}$ Pacific Northwest National Laboratory, Richland, Washington 99354, USA \\ ${ }^{3}$ Department of Physics and Astronomy, University of South Carolina, Columbia, South Carolina 29208, USA \\ ${ }^{4}$ Oak Ridge National Laboratory, Oak Ridge, Tennessee 37830, USA \\ ${ }^{5}$ National Research Center "Kurchatov Institute" Institute for Theoretical and Experimental Physics, Moscow 117218, Russia \\ ${ }^{6}$ Department of Physics, University of South Dakota, Vermillion, South Dakota 57069, USA \\ ${ }^{7}$ Joint Institute for Nuclear Research, Dubna 141980, Russia \\ ${ }^{8}$ Department of Physics, Duke University, Durham, North Carolina 27708, USA \\ ${ }^{9}$ Triangle Universities Nuclear Laboratory, Durham, North Carolina 27708, USA \\ ${ }^{10}$ Department of Physics and Astronomy, University of North Carolina, Chapel Hill, North Carolina 27514, USA \\ ${ }^{11}$ Nuclear Science Division, Lawrence Berkeley National Laboratory, Berkeley, California 94720, USA \\ ${ }^{12}$ South Dakota School of Mines and Technology, Rapid City, South Dakota 57701, USA \\ ${ }^{13}$ Los Alamos National Laboratory, Los Alamos, New Mexico 87545, USA \\ ${ }^{14}$ Department of Physics and Astronomy, University of Tennessee, Knoxville, Tennessee 37916, USA \\ ${ }^{15}$ Research Center for Nuclear Physics, Osaka University, Ibaraki, Osaka 567-0047, Japan \\ ${ }^{16}$ Department of Physics, Princeton University, Princeton, New Jersey 08544, USA \\ ${ }^{17}$ Department of Physics, North Carolina State University, Raleigh, North Carolina 27695, USA \\ ${ }^{18}$ Department of Physics, Massachusetts Institute of Technology, Cambridge, Massachusetts 02139, USA \\ ${ }^{19}$ Department of Physics, Engineering Physics and Astronomy, Queen's University, Kingston, Ontario K7L 3N6, Canada \\ ${ }^{20}$ Max-Planck-Institut für Physik, München 80805, Germany \\ ${ }^{21}$ Physik Department, Technische Universität, München 85748, Germany
}

(Received 30 January 2018; revised manuscript received 23 April 2018; published 25 May 2018)

\begin{abstract}
The MAJORANA DEMONSTRATOR is an ultralow-background experiment searching for neutrinoless doublebeta decay in ${ }^{76} \mathrm{Ge}$. The heavily shielded array of germanium detectors, placed nearly a mile underground at the Sanford Underground Research Facility in Lead, South Dakota, also allows searches for new exotic physics. Free, relativistic, lightly ionizing particles with an electrical charge less than $e$ are forbidden by the standard model but predicted by some of its extensions. If such particles exist, they might be detected in the MAJORANA DEMONSTRATOR by searching for multiple-detector events with individual-detector energy depositions down to $1 \mathrm{keV}$. This search is background-free, and no candidate events have been found in 285 days of data taking. New direct-detection limits are set for the flux of lightly ionizing particles for charges as low as $e / 1000$.
\end{abstract}

DOI: 10.1103/PhysRevLett.120.211804

Published by the American Physical Society under the terms of the Creative Commons Attribution 4.0 International license. Further distribution of this work must maintain attribution to the author(s) and the published article's title, journal citation, and DOI. Funded by SCOAP .
Lightly ionizing particles (LIPs) are hypothetical particles for which the electromagnetic interaction is suppressed compared to particles like charged hadrons and leptons. A particle with a charge $q=e / f$ that is reduced by a factor $f$ relative to the electron charge $e$ is expected to 
have weaker electromagnetic interactions than standard singly charged particles. These particles are often referred to as milli- or minicharged particles (MCPs) in the literature. In this Letter, we refer to them as LIPs, since this designation describes the energy loss phenomenology related to a class of detection techniques. The term LIPs includes MCPs, since their signature would be diminished ionization, but it does not preclude other possible particles.

The standard model (SM) of particle physics does not include free fractionally charged particles [1], since the quarks are bound within hadrons and do not exist as free particles. However, the SM is known to be incomplete, since it cannot explain the nature of dark matter or dark energy. Unbound quarks, noninteger-charged bound states of quarks, or new leptons with a fractional charge are a few possible candidates with LIP character that occur in proposed extensions of the SM. There are a variety of theories that permit an MCP including, for example, a fermion singlet $[2,3]$, an additional mirror $\mathrm{U}(1)$ paraphoton that can mix with the photon [4], neutrinos with electromagnetic couplings [5], vector particles [6], dark constituents bound to atoms [7], charge quantization [8-11], or composite MCPs [12]. The phenomenology of these models and their variants is very broad, justifying a variety of search techniques and leading to a rich experimental literature.

Although the masses of these particles can lie above the reach of current accelerators, experimental constraints on masses and charges of MCPs have been derived from fixed target accelerators [13-20], colliders [21-28], stellar models $[3,29-31]$, the cosmic microwave background [29,30, 32-37], big-bang nucleosynthesis [30], Supernova 1987A $[30,38]$, neutron stars $[39,40]$, pulsars and gamma ray bursts [41], galaxy clusters [42], the Lamb shift [29,43,44], dark cosmic ray searches [45], positronium decay [46], reactor neutrinos [47,48], and the $\mu$ magnetic moment [29]. An early levitation experiment [49] found an indication for the existence for fractional charges that was not confirmed by following efforts [50,51]. Millikan's method is a longstanding technique to search for fractional charges [52], combining the advantage of large probe sizes and high counting statistics. Brownian motion, however, limits this method's sensitivity [53]. Direct searches for LIPs, including MACRO [54,55], Kamiokande-II [56], and LSD [57] placed stringent limits on the LIP flux for $0.4<f<6$. The Cryogenic Dark Matter search (CDMS) experiment $[58,59]$ placed limits on exotic particles with $f<200$ using a direct search technique. A 2009 review [60] summarizes the experimental state of the field prior to the results of CDMS in 2010. References [3,61] provide a broad list of references, give a recent overview of the results over the past decade, and discuss the mass-charge parameter space. Here, we describe an improved direct search for such particles.

The Majorana Demonstrator $[62,63]$ is located at a depth of $4850 \mathrm{ft}$ at the Sanford Underground Research

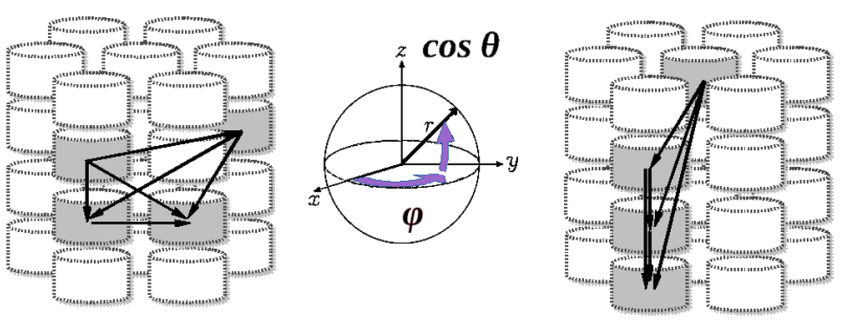

FIG. 1. Sketch of the detector arrangement and the vectors used in the background rejection cut. The gray shading indicates four detectors that triggered in this example. Left: Vectors connecting the detector centers for a sample noise or background event, which do not point to a common location. Middle: Definition of the angles used in the tracking algorithm. Right: For a simulated LIP, the variation of directions $(\Delta \cos \theta$ and $\Delta \phi)$ is smaller.

Facility [64]. In addition to its primary goal of searching for neutrinoless double-beta decay, its ultralow-background configuration permits additional physics studies including searches for dark matter, axions, and exotic physics [65]. Two modules contain $44.1 \mathrm{~kg}$ of high-purity germanium detectors, $29.7 \mathrm{~kg}$ of which are enriched to $88 \%{ }^{76} \mathrm{Ge}$. Fiftyeight detector units are installed in strings of three, four, or five detectors. The detector masses, diameters, and heights range from 0.5 to $1 \mathrm{~kg}, 6$ to $8 \mathrm{~cm}$, and 3 to $4 \mathrm{~cm}$, respectively. A sketch of the setup can be seen in Fig. 1, and a detailed description can be found in Ref. [62]. The MAJORANA DEMONSTRATOR detectors are 3-5 times thicker than those used in CDMS, providing a higher sensitivity to lower-energy deposits per crossing and hence higher values of $f$ at comparable energy thresholds. The low thresholds, excellent energy resolution, reduced electronic noise, and pulse shape characteristics of the $p$-type point contact detectors [66-69] allow a competitive LIP search based on the DEMONSTRATOR data.

The analysis presented here includes data taken from June 2015 until March 2017. Excluding calibration, commissioning, and blind data, the analyzed data include 285 days of live time, of which 121 days were taken with both modules operating in the final DEMONSTRATOR configuration [63]. This corresponds to a total exposure of $4993 \mathrm{~kg}$ days for all detectors. Physics runs are typically one hour long. Since the set of operable detectors and their respective thresholds changed over the course of data taking, our simulation mirrored the changing conditions on a run-by-run basis. For several runs, the threshold was increased to avoid noise introduced by external work during the construction phase.

The flux $[\Phi(f)]$ of LIPs through the detector array is given as

$$
\Phi(f)=\frac{n}{\sum_{i} \sum_{m} A_{i, m} \epsilon_{i, m} t_{i} \Omega_{i, m}},
$$

where $n$ is the number of detected interactions. For zero candidates, an upper bound on $\Phi$ can be set using the 
method of Feldman and Cousins in Ref. [70]. The sum index $i$ is over data runs, and index $m$ is over the multiplicity values considered for LIP candidates. The multiplicity is defined as the number of detectors with signals above the threshold within a $4-\mu$ s-long coincidence window. We consider events with $m=4,5$, and 6 . The length of a run is given by its dead-time corrected live time $\left(t_{i}\right)$. The detection efficiency $(\epsilon)$ depends on each detector's threshold and the geometry of the active detectors, both of which vary run by run. On average, $70 \%$ of the detectors are operable. The detection threshold was estimated by analyzing the baseline noise of each recorded waveform and verified in special forced trigger data. The detector baseline traces are processed with a trapezoidal filter. From the distribution of the integrated values of the flattop, we can estimate the energy at which we would detect events with a $99.7 \%$ or greater probability. For the majority of the runs, the individual thresholds are between 0.8 and $2 \mathrm{keV}$. The surface area $\left(A_{i, m}\right)$ for an incident LIP is taken as the end cap area of the smallest detector crossed. For the Demonstrator detectors, $A=30-37 \mathrm{~cm}^{2}\left( \pm 1 \mathrm{~cm}^{2}\right)$. The MAGE [71,72] framework, based on GEANT4 [73], was used to estimate $A_{i, m}$ and the solid angle $\Omega$ for each run. Simulated noninteracting particles were used as a proxy for LIPs and propagated through the array with varying angles of incidence. Since the path length through detectors depends on the LIP trajectory angle through the array, the efficiency is a function of the incident angle therefore depends on the impinging flux distribution. CDMS assumed an isotropic distribution from above [59]. We present results for that same distribution for comparison as well as results for a $\cos ^{2} \theta$ distribution, where $\theta$ is the polar angle. The latter function is a proxy for particles created in the upper atmosphere [60]. For $m=4$ events, the average solid angle is $\sim 2.4 \mathrm{sr}(1.5 \mathrm{sr})$ for a uniform flux from above $\left(\cos ^{2} \theta\right.$ distribution). The exact number varies for each run. Larger $m$ 's have a smaller number of possible detector combinations and, hence, smaller $\Omega$. For $m=5$ and 6 , the average solid angles are $1(0.6)$ and $0.06 \mathrm{sr}(0.02 \mathrm{sr})$, respectively.

For large $f$, LIPs interact potentially only once in a detector (cf. Fig. 2), leading to large energy-deposit fluctuations. Following Refs. [59,74], we calculate the expected energy-loss distribution based on the singleinteraction energy loss. The photoabsorption ionization model [75] was used to calculate the interaction cross section. This probability distribution function (PDF) for the single-interaction energy loss is convolved with itself $N$ times to derive the PDF for $N$ such interactions [76]; cf. Fig. 3. The number of interactions per unit path length through a detector was calculated using the approach of Ref. [77]. The result is a function of $f$ as shown in Fig. 2. The expected energy deposited as a function of the track length and $f$ is shown in Fig. 4. The probability that a LIP with $f$ deposits enough energy to exceed the detector

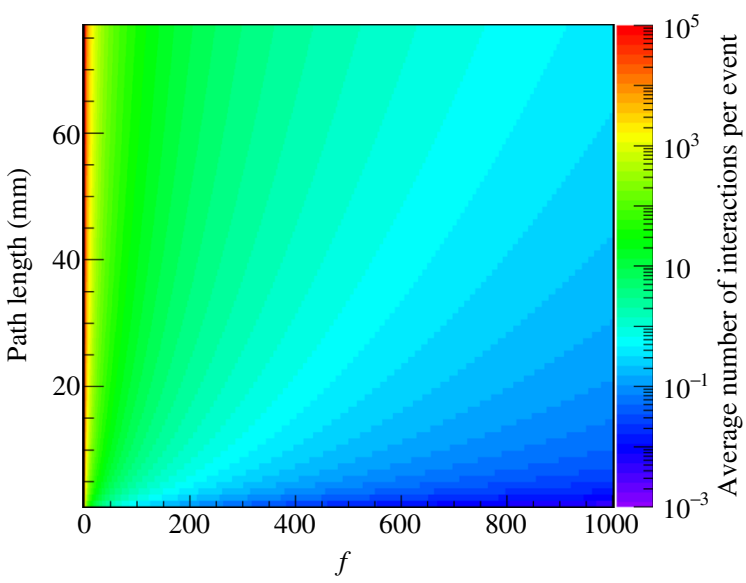

FIG. 2. Average number of interactions for one LIP event in germanium as a function of the path length and the parameter $f=e / q$.

threshold is calculated for simulated events. The total efficiency is the product of these individual detector probabilities.

For each run and detector, the data acquisition threshold is applied in combination with the simulation, resulting in a run-dependent detection efficiency for LIPs with a given $m$ and trajectory. The simulated efficiency distributions also take into account inoperable channels and exclude them from the analysis.

Two factors give non-negligible contributions to the uncertainty of the efficiency $\epsilon$. One is the uncertainty in the traversed-detector path length that determines the number of interactions. This results from uncertainty in the thickness of the dead layer at the outer surface of each detector. The other factor is that the detectors have a finite energy resolution. Both effects contribute to the uncertainty in the probability that a LIP energy deposit will be above the threshold, especially for large $f$ and small energy depositions. In order to estimate systematic uncertainties,

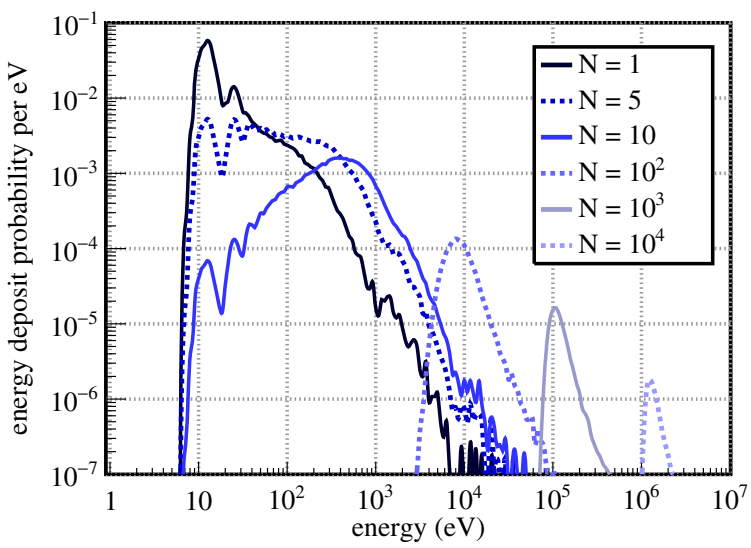

FIG. 3. Expected energy loss for several numbers of interaction $N$. All curves are calculated Poisson-weighted convolutions of the single interaction distribution. 


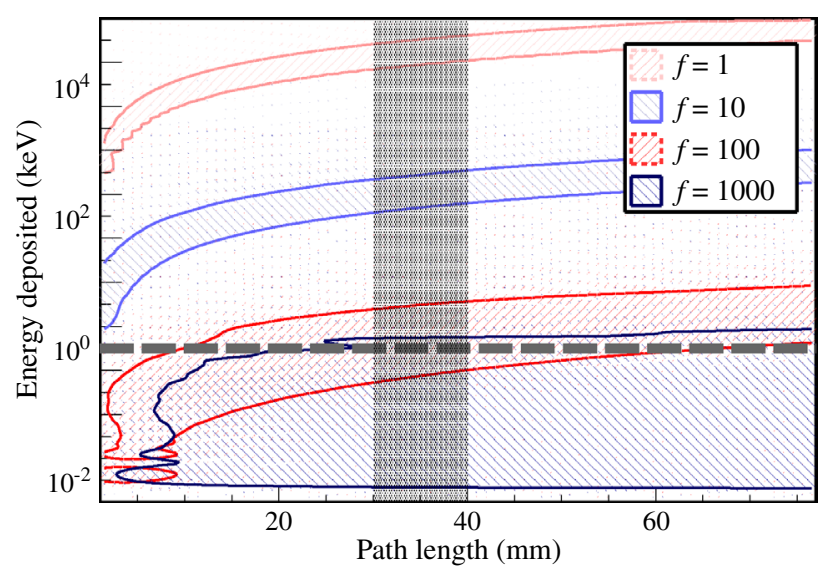

FIG. 4. Energy depositions of simulated events with different path lengths in germanium. The colored bands indicate the $90 \%$ enclosure of all events for four different values of the denominator $f$, respectively. The horizontal dark gray dashed line indicates a $1 \mathrm{keV}$ threshold, and the vertical gray area indicates the average detector thickness.

we analyzed the simulated efficiencies 100 times for each individual run, varying the track length $l$ inside each detector traversed and the energy resolution. The values were drawn from Gaussian distributions around the mean value of each parameter, with widths $\sigma_{l}= \pm 1 \mathrm{~mm}$, $\sigma_{n}=\sqrt{n}$, and $\mathrm{FWHM}_{E}=0.2 \mathrm{keV}$, respectively. The energy resolution value corresponds to the FWHM below $10 \mathrm{keV}$ in the Demonstrator [65]. Finally, all the efficiencies for a given data set and multiplicity $m$ are combined in one histogram. In Fig. 5, the distribution of efficiencies for $m=4$ events is drawn. The width of the distribution for each value of $f$ is used as the systematic uncertainty. This conservative approach allows us to show that our sensitivity is mostly independent of short-lived variations in detector settings.

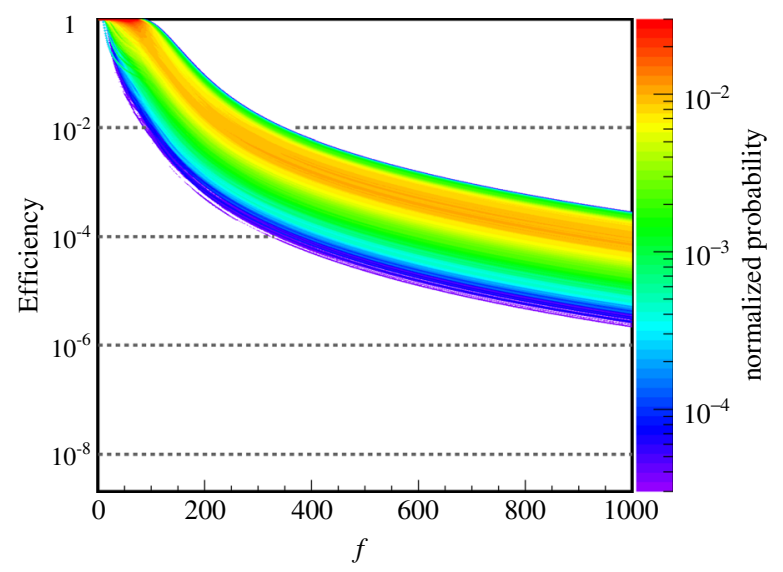

FIG. 5. Detection efficiency for $m=4$ events for one data set as a function of $f$, the factor by which the charge of the particle is smaller than the elementary charge. For each individual $f$, the probabilities of the detection efficiencies are given by the color scale.
In each detector within the DEMONSTRATOR, a fair number of nonphysics events contribute to the low-energy backgrounds. These include noise, microphonics during nitrogen fills, and pulser cross talk. A multiplicity requirement of $4 \leq m \leq 6$ eliminates the majority $(\approx 97 \%)$ of these without significant additional analysis. In addition, a onesecond anticoincidence time with the muon veto of the DEMONSTRATOR excludes cosmogenic background. All events surviving events from the 285 days of live time (corresponding to $4993 \mathrm{~kg}$ days) are depicted in Fig. 6. There is no requirement on the geometric arrangement allowing us to greatly increase $\Omega$, and therefore sensitivity, relative to the CDMS experiment. In other words, instead of searching only for particles from above, we also search for LIPs that traverse multiple strings.

Because of the variation of detector sizes and variety of possible LIP trajectories, it is impossible to include a CDMS-like energy consistency requirement; the path lengths in different detectors are not necessarily comparable. To reduce the remaining background within the high$m$ sample, a tracking algorithm was applied. Each candidate event is compared to the simulated signature of a LIP. A LIP will traverse the array in a straight line, and vectors connecting pairs of triggered detectors (see the rightmost panel in Fig. 1) should all point roughly to the same direction on an imaginary sphere surrounding the array. Since the exact location of the interaction within the detector is unknown, the center of the detectors is used as the start and end point of each vector. The direction of these vectors can be described with two angles using spherical coordinates $\theta$ and $\phi$, depicted in Fig. 1.

Since their triggered detectors do not fall along a single track, events due to instrumental effects and internal

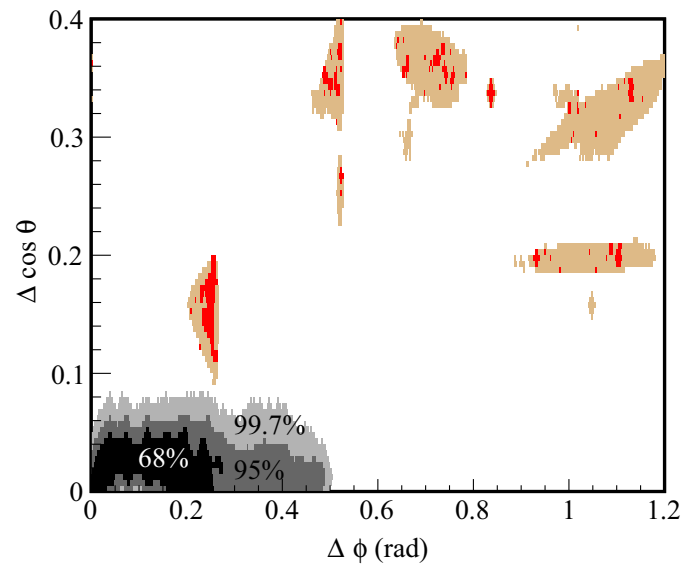

FIG. 6. Event-by-event spread in the two spherical angles (see Fig. 1) for Demonstrator data (brown) during the 285-day lifetime period. Events within a one-second coincidence with the muon veto [78] are shown in red. Almost 25000 events survive the basic multiplicity cut applied for this study. For simulated LIPs from an isotropic source, the regions that include $68 \%$ (black), 95\% (dark gray), and 99.7\% (used in analysis, light gray) of all events are shown. 
backgrounds will self-evidently have larger values of $\Delta \theta$ and $\Delta \phi$, the differences in $\theta$ and $\phi$ determined from different detector pairings in a single event. Distinguishing muons from LIPs with the tracking algorithm may seem more difficult; we can study such tracks by choosing events that are triggered in coincidence with the muon veto system. A minimally ionizing LIP with high $f$ $(>6)$ would not deposit enough energy to trigger the veto, which is made of 2-inch-thick plastic scintillator panels and has a trigger threshold of $1 \mathrm{MeV}$. For muon events, the particle shower accompanying the muon tends to trigger more than six detectors, or additional out-of-line detectors, as shown by the red muon veto-coincident events in Fig. 6. Simulations show that LIPs with $f>6$ do not produce significant showers, unlike muons. For $f=1,90 \%$ of the events are accompanied by a shower. For $f=6$, this number drops to only $7 \%$ and can be assumed to be close to zero for higher $f$. Therefore, the simulated LIP events show smaller spreads in $\theta$ and $\phi$ values than muon events. Since our analysis requires linelike shower-free events, we excluded limits below $f=6$ from our results.

A cut in $\Delta \theta$ and $\Delta \phi$, shown by the gray region in Fig. 6 , was chosen based on the LIP simulations. The efficiency for retaining a LIP candidate in the tracking algorithm is effectively unity with an uncertainty of less than $0.3 \%$, which is negligible compared to the other uncertainties. Restricting the multiplicities to $m=4,5$, or 6 events in the DEMONSTRATOR data, we find no LIP candidate events in the shaded area. Applying the Feldman and Cousins procedure [70], a value of 2.44 (90\% C.L.) is used as the upper limit for $n$ in Eq. (1).

Figure 7 displays the results as a function of $f$. For charges between $e / 6$ and $e / 30$, a limit of $2 \times 10^{-9}$ particles

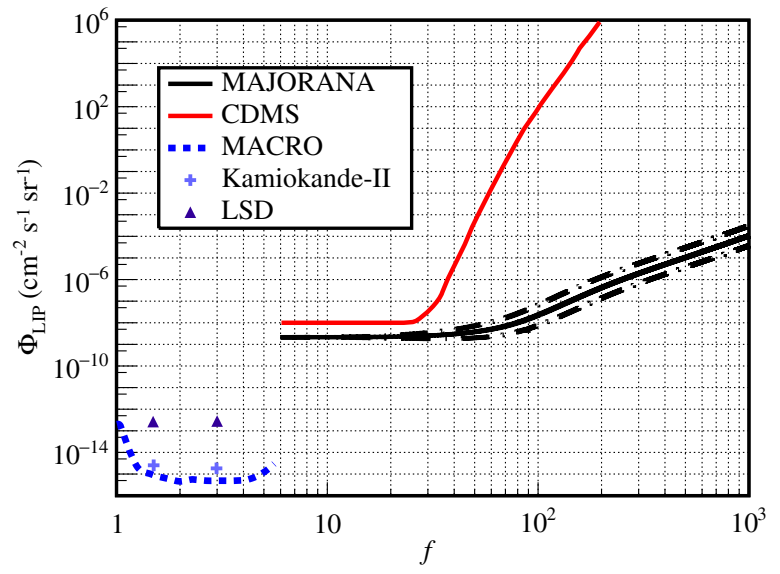

FIG. 7. LIP flux limit from above on the MAJORANA DEMONSTRATOR using a $90 \%$ confidence level (black line) and its $1 \sigma$ uncertainty bands (dashed black lines). Results from MACRO [55], Kamiokande-II [56], LSD [57], and CDMS [59] are shown as well. All limits assume an isotropic flux. As indicated in the text, a $\cos ^{2} \theta$ distribution of LIPs would result in a $38 \%$ less restrictive curve. per $\mathrm{cm}^{2} \mathrm{~s} \mathrm{sr}$ is found. A deviation from the minimally ionizing character $(\beta \gamma \sim 3)$ of the particle would result in a higher detection efficiency. Hence, the limits presented are conservative upper limits. Using the assumption that LIPs are impinging with a $\cos ^{2} \theta$ distribution would result in a slightly smaller detection efficiency and, therefore, in a limit that is about $38 \%$ above that of the isotropic model.

This work presents the first limits on massive relativistic particles with a fractional charge using the unique features of the Majorana Demonstrator. The large path length due to thick detectors in combination with the low thresholds allows for a sensitivity down to $1 / 1000$ of an elementary charge. These are the first results for a nonaccelerator-based experiment on the natural flux of lightly ionizing particles with charges less than $e / 200$ and an improvement of the existing limits for charges between $e / 6$ and $e / 200$. The results presented will help to exclude certain models or at least restrict their parameter space, e.g., for the millicharged dark matter presented in Ref. [45].

This material is based upon work supported by the U.S. Department of Energy, Office of Science, Office of Nuclear Physics under Awards No. DE-AC02-05CH11231, No. DE-AC05-00OR22725, No. DE-AC05-76RL0130, No. DE-AC52-06NA25396, No. DE-FG02-97ER41020, No. DE-FG02-97ER41033, No. DE-FG02-97ER41041, No. DE-SC0010254, No. DE-SC0012612, No. DESC0014445, and No. DE-SC0018060. We acknowledge support from the Particle Astrophysics Program and Nuclear Physics Program of the National Science Foundation through Grants No. MRI-0923142, No. PHY-1003399, No. PHY-1102292, No. PHY1206314, and No. PHY-1614611. We gratefully acknowledge the support of the U.S. Department of Energy through the LANL/LDRD Program and through the PNNL/LDRD Program for this work. We acknowledge support from the Russian Foundation for Basic Research, Grant No. 15-0202919. We acknowledge the support of the Natural Sciences and Engineering Research Council of Canada, funding reference No. SAPIN-2017-00023, and from the Canada Foundation from Innovation John R. Evans Leaders Fund. We thank the Yamaha Science Foundation Japan for their support. This research used resources provided by the Oak Ridge Leadership Computing Facility at Oak Ridge National Laboratory and by the National Energy Research Scientific Computing Center, a DOE Office of Science User Facility. We thank our hosts and colleagues at the Sanford Underground Research Facility for their support.

\footnotetext{
*Present address: Centro de Investigaciones Energéticas, Medioambientales y Tecnológicas, CIEMAT 28040, Madrid, Spain.

Corresponding author. massarczyk@lanl.gov
} 
${ }^{\ddagger}$ Present address: Los Alamos National Laboratory, Los Alamos, New Mexico, USA.

[1] P. Langacker and S.-Y. Pi, Phys. Rev. Lett. 45, 1 (1980).

[2] L. Okun, M. Voloshin, and V. Zakharov, Phys. Lett. B 138, 115 (1984).

[3] N. Vinyoles and H. Vogel, J. Cosmol. Astropart. Phys. 03 (2016) 002.

[4] B. Holdom, Phys. Lett. 166B, 196 (1986).

[5] R. Foot, G. Joshi, H. Lew, and R. Volkas, Mod. Phys. Lett. A 05, 95 (1990).

[6] E. Gabrielli, L. Marzola, M. Raidal, and H. Veermäe, J. High Energy Phys. 08 (2015) 150.

[7] J. M. Cline, Z. Liu, and W. Xue, Phys. Rev. D 85, 101302 (2012).

[8] A. Y. Ignatiev, V. A. Kuzmin, and M. E. Shaposhnikov, Phys. Lett. B 84, 315 (1979).

[9] X.-G. Wen and E. Witten, Nucl. Phys. B261, 651 (1985).

[10] A. Schellekens, Phys. Lett. B 237, 363 (1990).

[11] K. S. Babu and R. N. Mohapatra, Phys. Rev. D 41, 271 (1990).

[12] C. Kouvaris, Phys. Rev. D 88, 015001 (2013).

[13] J. Aubert et al., Phys. Lett. B 133, 461 (1983).

[14] F. Bergsma et al., Z. Phys. C 24, 217 (1984).

[15] E. Golowich and R. W. Robinett, Phys. Rev. D 35, 391 (1987)

[16] Y. D. He and P. B. Price, Phys. Rev. C 44, 1672 (1991).

[17] G. Hüntrup, D. Weidmann, S. E. Hirzebruch, E. Winkel, and W. Heinrich, Phys. Rev. C 53, 358 (1996).

[18] D. Ghosh et al., Fiz. B 5, 135 (1996).

[19] A. A. Prinz et al., Phys. Rev. Lett. 81, 1175 (1998).

[20] D. E. Soper, M. Spannowsky, C. J. Wallace, and T. M. P. Tait, Phys. Rev. D 90, 115005 (2014).

[21] F. Abe et al. (CDF Collaboration), Phys. Rev. D 46, R1889 (1992).

[22] D. Buskulic et al., Phys. Lett. B 303, 198 (1993).

[23] R. Akers et al., Z. Phys. C 67, 203 (1995).

[24] P. Abreu et al., Phys. Lett. B 396, 315 (1997).

[25] D. Acosta et al., Phys. Rev. Lett. 90, 131801 (2003).

[26] G. Abbiendi et al., Phys. Lett. B 572, 8 (2003).

[27] J. Jaeckel, M. Jankowiak, and M. Spannowsky, Phys. Dark Universe 2, 111 (2013).

[28] S. Chatrchyan et al. (CMS Collaboration), Phys. Rev. D 87, 092008 (2013).

[29] M. I. Dobroliubov and A. Y. Ignatiev, Phys. Rev. Lett. 65, 679 (1990).

[30] S. Davidson, S. Hannestad, and G. Raffelt, J. High Energy Phys. 05 (2000) 003.

[31] J. L. Feng, J. Smolinsky, and P. Tanedo, Phys. Rev. D 93, 115036 (2016).

[32] M. Ahlers, Phys. Rev. D 80, 023513 (2009).

[33] C. Burrage et al., J. Cosmol. Astropart. Phys. 11 (2009) 002.

[34] S. L. Dubovsky, D. S. Gorbunov, and G. I. Rubtsov, Pis'ma Zh. Eksp. Teor. Fiz. 79, 3 (2004) [JETP Lett. 79, 1 (2004)].

[35] Z. Berezhiani and A. Lepidi, Phys. Lett. B 681, 276 (2009).

[36] H. Vogel and J. Redondo, J. Cosmol. Astropart. Phys. 02 (2014) 029.

[37] A. D. Dolgov and A. S. Rudenko, J. Exp. Theor. Phys. 124, 564 (2017).
[38] R. N. Mohapatra and I. Z. Rothstein, Phys. Lett. B 247, 593 (1990).

[39] X. Huang, X.-P. Zheng, W.-H. Wang, and S.-Z. Li, Phys. Rev. D 91, 123513 (2015).

[40] M. Korwar and A. M. Thalapillil, arXiv:1709.07888.

[41] A. K. Kvam and D. C. Latimer, arXiv:1412.0708.

[42] K. Kadota, T. Sekiguchia, and H. Tashirob, arXiv:1602 04009

[43] S. Davidson, B. Campbell, and D. C. Bailey, Phys. Rev. D 43, 2314 (1991).

[44] M. Glück, S. Rakshit, and E. Reya, Phys. Rev. D 76, 091701 (2007).

[45] P.-K. Hu, A. Kusenko, and V. Takhistov, Phys. Lett. B 768, 18 (2017).

[46] A. Badertscher, P. Crivelli, W. Fetscher, U. Gendotti, S. N. Gninenko, V. Postoev, A. Rubbia, V. Samoylenko, and D. Sillou, Phys. Rev. D 75, 032004 (2007).

[47] S. N. Gninenko, N. V. Krasnikov, and A. Rubbia, Phys. Rev. D 75, 075014 (2007).

[48] J.-W. Chen, H.-C. Chi, H.-B. Li, C.-P. Liu, L. Singh, H. T. Wong, C.-L. Wu, and C.-P. Wu, Phys. Rev. D 90, 011301 (2014)

[49] G. S. LaRue, J. D. Phillips, and W. M. Fairbank, Phys. Rev. Lett. 46, 967 (1981).

[50] P. Smith, G. J. Homer, J. D. Lewin, H. E. Walford, and W. G. Jones, Phys. Lett. B 153, 188 (1985).

[51] D. C. Moore, A. D. Rider, and G. Gratta, Phys. Rev. Lett. 113, 251801 (2014).

[52] P. C. Kim, E. R. Lee, I. T. Lee, M. L. Perl, V. Halyo, and D. Loomba, Phys. Rev. Lett. 99, 161804 (2007).

[53] V. Halyo, P. Kim, E. R. Lee, I. T. Lee, D. Loomba, and M. L. Perl, Phys. Rev. Lett. 84, 2576 (2000).

[54] M. Ambrosio et al., Phys. Rev. D 62, 052003 (2000).

[55] M. Ambrosio et al., arXiv:hep-ex/0402006.

[56] M. Mori et al., Phys. Rev. D 43, 2843 (1991).

[57] M. Aglietta et al., Astropart. Phys. 2, 29 (1994).

[58] Z. Ahmed et al. (CDMS II Collaboration), Science 327, 1619 (2010).

[59] R. Agnese et al. (CDMS Collaboration), Phys. Rev. Lett. 114, 111302 (2015).

[60] M. L. Perl, E. R. Lee, and D. Loomba, Annu. Rev. Nucl. Part. Sci. 59, 47 (2009).

[61] A. Haas, C. S. Hill, E. Izaguirre, and I. Yavin, Phys. Lett. B 746, 117 (2015).

[62] N. Abgrall et al., Adv. High Energy Phys. 2014, 1 (2014).

[63] C. Aalseth et al., Phys. Rev. Lett. 120, 132502 (2018).

[64] J. Heise, J. Phys. Conf. Ser. 606, 012015 (2015).

[65] N. Abgrall et al. (MAJORAnA Collaboration), Phys. Rev. Lett. 118, 161801 (2017).

[66] P. N. Luke, F. S. Goulding, N. W. Madden, and R. H. Pehl, IEEE Trans. Nucl. Sci. 36, 926 (1989).

[67] P. S. Barbeau, J. I. Collar, and O. Tench, J. Cosmol. Astropart. Phys. 09 (2007) 009.

[68] E. Aguayo et al., arXiv:1109.6913.

[69] R. Cooper, D. C. Radford, K. Lagergren, J. F. Colaresi, L. Darken, R. Henning, M. G. Marino, and K. M. Yocum, Nucl. Instrum. Methods Phys. Res., Sect. A 629, 303 (2011).

[70] G. J. Feldman and R. D. Cousins, Phys. Rev. D 57, 3873 (1998). 
[71] M. Bauer et al., J. Phys. Conf. Ser. 39, 362 (2006).

[72] M. Boswell et al., IEEE Trans. Nucl. Sci. 58, 1212 (2011).

[73] S. Agostinelli et al., Nucl. Instrum. Methods Phys. Res., Sect. A 506, 250 (2003).

[74] K. B. Prasad, Ph.D. thesis, Texas A\&M University, 2013.
[75] W. Allison and J. Cobb, Annu. Rev. Nucl. Part. Sci. 30, 253 (1980).

[76] H. Bichsel, Nucl. Instrum. Methods Phys. Res., Sect. A 562, 154 (2006).

[77] H. Bichsel, Rev. Mod. Phys. 60, 663 (1988).

[78] N. Abgrall et al., Astropart. Phys. 93, 70 (2017). 